\title{
Hepatitis-C-Therapie als Primärprävention?
}

\author{
Dank Präventionsmassnahmen sind in der Schweiz wie in den meisten westlichen \\ Ländern die HIV-Ansteckungen bei Drogenkonsumenten deutlich zurückgegangen - \\ ganz anders verhält es sich bei den Hepatitis-C-Infektionen. Am «2nd International \\ Symposium on Hepatitis Care in Substance Users» widmeten sich in Brüssel zwei- \\ hundert Kolleginnen und Kollegen diesem Phänomen.
}

Philip Bruggmann

Chefarzt Innere Medizin Arud Zürich
Korrespondenz:

Dr. med. Philip Bruggmann Arbeitsgemeinschaft für risikoarmen Umgang mit Drogen Arud Konradstrasse 1 CH-8005 Zürich p.bruggmann(at)arud.ch www.arud.ch

\section{HIV ist nicht gleich HCV}

Mit Harm-Reduction-Massnahmen (Abgabe von sauberen Spritzen, Nadeln und Drogenzubereitungsmaterialien) konnten in den letzten zwanzig Jahren die Inzidenz und Prävalenz von HIV bei Drogenkonsumierenden deutlich gesenkt werden.

Im Gegensatz dazu blieben die Ansteckungsraten und auch die Zahl von bestehenden Infektionen von Hepatitis C nahezu konstant. Dies ist ein weltweit weitverbreitetes Phänomen und war am «2nd International Symposium on Hepatitis Care in Substance Users» (INHSU-Symposium, siehe auch www.inhsu. com) eines der meistdiskutierten Themen unter den zweihundert Ärzten und Fachleuten aus zwanzig Ländern. Als mögliche Gründe für den unterschiedlichen Präventionseffekt wurden die tieferen Behandlungsraten wegen fehlender nationaler Programme und die höhere Infektiosität und das längere Überleben des Hepatitis-C-Virus an der Luft genannt. höher als bei unseren europäischen Nachbarn. Es fehlt ein national koordiniertes Vorgehen, das auf der Aufklärungskampagne von Infodrog (www.hepch.ch) aufbaut und ein flächendeckendes, den Bedürfnissen der Zielgruppe angepasstes Screening- und Behandlungsangebot vorsieht. Im Gegensatz zu Syphilis und HIV wurde Hepatitis C vom Bundesamt für Gesundheit (BAG) bis heute nicht der notwendige Stellenwert eingeräumt.

\section{Hohe Folgekosten für die Zukunft}

Die am INHSU-Symposium in Brüssel präsentierten Daten sollten hellhörig machen: Studien zeigen, dass bei Drogenkonsumierenden mit einer chronischen Hepatitis-C-Infektion die Morbidität (Zirrhose) und die Mortalität mit zunehmenden Alter exponentiell ansteigt. Eine besonders starke Zunahme von Zirrhose und leberbedingter Mortalität ist gemäss dieser Untersuchungen im Alter von 45 bis 50 Jahren zu er-

\section{«In der Schweiz wird an verschiedenen Orten bereits eine starke Zunahme von leberbedingten Hospitalisationen und Todesfällen beobachtet.»}

\section{Ungenutzte «Poleposition» der Schweiz}

Die Schweiz verfügt weltweit über die meisten Substitutionstherapieplätze und ein international viel beachtetes Behandlungskonzept für Drogenkonsumierende: Eine optimale Grundlage, diese Patienten auch bezüglich Infektionskrankheiten zu behandeln. Auf nationaler Ebene wurde jedoch die Chance bislang nicht wahrgenommen, der Hepatitis-C-Epidemie unter Drogenkonsumierenden Herr zu werden. Nirgends in Europa werden prozentual so viele Opiatabhängige substituiert und sind somit in einer strukturierten medizinischen Versorgung eingebunden. Und trotzdem sind in der Schweiz die Hepatitis-CBehandlungsraten bei Drogenkonsumierenden nicht warten. In vielen Schweizer Substitutionsinstitutionen liegt heute das Durchschnittsalter der Klienten bei gut 40 Jahren. In der Schweiz wird an verschiedenen Orten bereits eine starke Zunahme von leberbedingten Hospitalisationen und Todesfällen beobachtet. Die damit verbundenen gesundheitsökonomischen Folgen sind vorauszusehen.

\section{Antivirale Therapie als Primärprävention}

Bei HIV-Infektionen ist die Indikation zur antiviralen Therapie zwecks Verhütung der Weiterverbreitung des Virus schon länger ein Thema. Jetzt wird auch im Hepatitis-C-Bereich diese Massnahme diskutiert, zumindest für die grosse Risikogruppe der Drogenkon- 
sumierenden. Am INHSU-Symposium in Brüssel wurde eine Studie aus Grossbritannien vorgestellt, der eine mathematische Modellrechnung zugrunde liegt. Sie kommt zum Schluss, dass mittels realistischer Therapieraten die Prävalenz der Hepatitis C massgeblich gesenkt werden kann: Für eine chronische Hepatitis-C-Prävalenz, wie sie bei rund 40 Prozent der Drogenkonsumierenden vorliegt, könnte mit einer Therapierate von jährlich 20 HCV-Therapien pro 1000 Drogenkonsumierende innerhalb von 10 Jahren eine relative Reduktion der Prävalenz um 30 Prozent erreicht werden. Die Therapierate kann insofern als realistisch bezeichnet werden, als sie der verglichen. Bei einer HCV-Prävalenz von 40 Prozent in der Drogenpopulation (dies entspricht der aktuellen Situation in der Schweiz) ist die Therapie von Drogenkonsumierenden kosteneffizienter als die Therapie von anderen HCV-Patienten und zudem günstiger als Nichttherapieren (gemessen am Verhältnis zusätzlich gewonnener Lebensjahre und -qualität und den daraus entstehenden Mehrkosten).

\section{Modelle der integrierten Versorgung}

In verschiedenen Beiträgen aus diversen Ländern wurde die Möglichkeit einer in die suchtmedizinische Grundversorgung integrierte Hepatitis-C-Therapie

\section{Ein rasches, national koordiniertes Vorgehen auf mehreren Ebenen ist erforderlich, von der Aufklärung über Screening bis zur Therapie.}

aktuellen Aufnahmefrequenz von HCV-Therapien in den Zentren für Suchtmedizin der Arud in Zürich und Horgen entspricht.

\section{Wer soll zahlen?}

Eine Hepatitis-C-Therapie ist nicht billig. Wer soll die Kosten gesteigerter Therapieraten bezahlen? Auch dazu wurden in Brüssel Daten geliefert, wiederum belegt mit einer mathematischen Modellrechnung. Unter Berücksichtigung von möglichen Reinfektionen wurde die Kosteneffizienz einer Hepatitis-C-Therapie bei Drogenkonsumierenden und bei HCV-Patienten, die sich nicht über den Drogenkonsum angesteckt haben, mit dem Zustand einer Nichttherapie

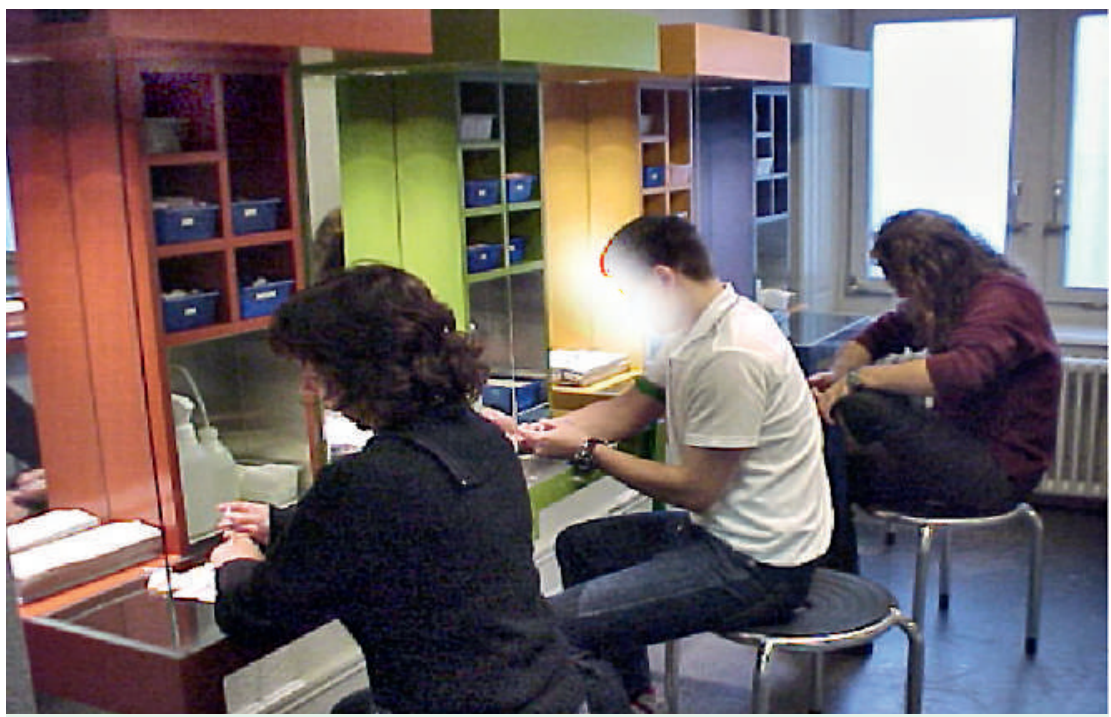

Trotz einer ausgeprägten medizinischen Versorgung ist es noch nicht gelungen, die Hepatitis-CAusbreitung bei Drogenkonsumierenden einzudämmen. diskutiert. Besonders betont wurde die Wichtigkeit von speziell geschultem Pflegepersonal bei Aufklärung, Vorbereitung zur Therapie und Begleitung durch die Behandlung. Auch der multidisziplinäre Ansatz mit raschem und strukturiertem Hinzuziehen von verschiedenen Fachspezialisten (Psychiater, Sozialarbeiter, Dermatologe usw.) wurde als unerlässlich bewertet. Eines der Referate zeigte auf, wie wichtig eine optimierte suchtmedizinische Behandlung respektive eine ausreichend dosierte Substitution für den positiven Ausgang einer Hepatitis-C-Therapie bei Drogenkonsumierenden ist. Voraussetzung für den Erfolg solcher Modelle, wie sie auch immer im Detail aussehen, ist ein fundiertes basales Fachwissen aller involvierten Fachrichtungen durch gegenseitige Schulungen.

\section{Nationaler Hepatitis-C-Plan}

Die grassierende Hepatitis-C-Epidemie vor allem unter Drogenkonsumierenden erfordert ein rasches, national koordiniertes Vorgehen auf der ganzen Linie: von der Aufklärung über Screening bis zur Therapie. Die Schweiz hat die besten Voraussetzungen dazu, ist sie doch weltweit führend in der Versorgung von Drogenabhängigen. Das Ziel sollte sein, die heute bestehenden, sehr gut funktionierenden Modelle der umfassenden Versorgung in den einzelnen Praxen und Institutionen auf das ganze Land auszudehnen.

\section{Referenzen}

- Abstracts und Zusammenfassungen aller Referate und Poster vom 2nd International Symposium on Hepatitis Care in Substance Users in Suchtmed. 2011;13(4):154ff.

- www.ecomed-medizin.de/suchtmedizin 